\title{
Atrial fibrillation risk factors in patients with ischemic stroke
}

Paweł Wańkowicz, Przemysław Nowacki, Monika Gołąb-Janowska

Department of Neurology, Pomeranian Medical University, Szczecin, Poland

Submitted: 5 January 2019

Accepted: 27 February 2019

Arch Med Sci 2021; 17 (1): 19-24

DOI: https://doi.org/10.5114/aoms.2019.84212

Copyright ( 2019 Termedia \& Banach

\section{Abstract}

Introduction: Atrial fibrillation (AF) is the most common heart arrhythmia. The condition is known to increase the risk of ischemic stroke (IS). Classical risk factors for the development of AF include advanced age, hypertension, diabetes mellitus, coronary heart disease and lipid metabolism disorders. Importantly, these are also recognized risk factors for ischemic stroke. Therefore, the purpose of this study was to investigate AF risk factors in patients with IS.

Material and methods: This is single-centre retrospective study which included 696 patients with acute ischemic stroke and nonvalvular atrial fibrillation and 1678 patients with acute ischemic stroke without atrial fibrillation.

Results: In this study we found - based on a univariable and multivariable logistic regression model - that compared to the patients with IS without AF, the group of patients which suffered from IS with nonvalvular atrial fibrillation (NVAF) had a higher proportion of patients who smoked cigarettes $(\mathrm{OR}=15.742, p<0.01 ; \mathrm{OR}=41.1, p<0.01)$, had hypertension $(\mathrm{OR}=5.161$, $p<0.01 ; \mathrm{OR}=5.666, p<0.01)$, history of previous stroke $(\mathrm{OR}=3.951$, $p<0.01 ; \mathrm{OR}=4.792, p<0.01)$, dyslipidemia $(\mathrm{OR}=2.312, p<0.01 ;$ $\mathrm{OR}=1.592, p<0.01)$, coronary heart disease $(\mathrm{OR}=2.306, p<0.01$; $\mathrm{OR}=1.988, p<0.01)$, a greater proportion of female patients $(\mathrm{OR}=1.717$, $p<0.01 ; \mathrm{OR}=2.095, p<0.01)$, higher incidence of diabetes mellitus $(\mathrm{OR}=1.341, p<0.01 ; \mathrm{OR}=1.261, p=0.106)$ and more patients in old age $(\mathrm{OR}=1.084, p<0.01 ; \mathrm{OR}=1.101, p<0.01)$.

Conclusions: Our study demonstrates a need for thorough and systematic monitoring of post-ischemic stroke patients in whom AF has not been detected and who display other important risk factors. Regardless of the stroke, these factors may be responsible for development of AF.

Key words: atrial fibrillation, risk factors, ischemic stroke.

\section{Introduction}

Atrial fibrillation (AF) is the most common heart arrhythmia [1]. The condition is known to increase the risk of ischemic stroke (IS) and peripheral embolism [2]. According to epidemiological studies, more than 5.6 million people in the USA are expected to suffer from AF by 2050, while in the European Union, the number of people over 55 with AF is expected to increase to 17.9 million between 2010 and 2060 [3, 4]. Classical risk factors for the development of AF include hypertension, advanced age, diabetes mellitus, coronary heart disease, and lipid metab-

\author{
Corresponding author: \\ Paweł Wańkowicz PhD \\ Department \\ of Neurology \\ Pomeranian \\ Medical University \\ 1 Unii Lubelskiej St \\ 71-252 Szczecin, Poland \\ Phone: +48 914253251 \\ E-mail: pawel.wankowicz@ \\ pum.edu.pl
}


olism disorders [5-7]. Importantly, these are also recognized risk factors for ischemic stroke [8-10]. Researchers have observed that patients with recurring IS often show evidence of untreated AF [11]. Brambatti et al. argue that the relationship between $\mathrm{AF}$ and thromboembolic events is related to many more factors than just the duration of AF. Some thromboembolic events may be due to AF-induced stagnation, some may be caused by chronic endothelial changes due to many previous AF episodes, or may be the result of co-existence of mechanisms other than AF for which AF is only a risk marker, e.g. co-existence of hypertension [12]. Therefore, the purpose of this study was to investigate AF risk factors in patients with IS.

\section{Material and methods}

We retrospectively examined 2374 patients with acute IS. The study group consisted of 696 patients (446 women and 250 men) with acute IS and NVAF and the control group had 1678 patients (855 women and 823 men) with acute IS without AF. All patients were hospitalized at the Department of Neurology in Szczecin.

Diagnosis of AF was based on medical history or ECG examination on admission to the Neurology Department or during hospitalization. IS was defined as sudden onset of a focal neurological deficit lasting more than $24 \mathrm{~h}$ by means of brain computed tomography and/or magnetic resonance imaging. Congestive heart failure diagnosis was based on signs and symptoms of low cardiac output based on medical charts [13]. Diabetes mellitus was defined as a fasting blood glucose level greater than $126 \mathrm{mg} / \mathrm{dl}$ after a minimum of 2 tests or glucose level greater than $200 \mathrm{mg} / \mathrm{dl}$ any time during the day [14]. Dyslipidemia was defined as the combination of serum cholesterol concentration $>190 \mathrm{mg} / \mathrm{dl}$, low-density lipoprotein (LDL) cholesterol $>115 \mathrm{mg} / \mathrm{dl}$, serum triglyceride concentration $>150 \mathrm{mg} / \mathrm{dl}$ and high-density lipoprotein (HDL) cholesterol $<40 \mathrm{mg} / \mathrm{dl}$ in males and $<45 \mathrm{mg} / \mathrm{dl}$ in females [15]. Hypertension was diagnosed when the blood pressure (BP) was $\geq 140 / 90 \mathrm{~mm} \mathrm{Hg}$ in repeated tests [16]. Coronary heart disease was determined by a previous history of myocardial infarction and angina pectoris. Smoker was defined as someone having smoked one or more cigarettes. Previous stroke was considered to be present if the medical charts included a stroke diagnosis. The $\mathrm{CHA}_{2} \mathrm{DS}_{2}$-VASc score (congestive heart failure/LV dysfunction (one point), hypertension (one point), age $\geq 75$ years (two points), diabetes mellitus (one point), stroke (two points), vascular disease (one point), age 65-74 years (one point), (female) sex category (one point) is a scale used to assess the risk of thromboembolic complications in patients with AF. It is an improved $\mathrm{CHADS}_{2}$ score, augmented with additional risk factors of thromboembolic complications. The $\mathrm{CHA}_{2} \mathrm{DS}_{2}$-VASC score was determined for each patient participating in this study. The Pomeranian Medical University Ethics Committee approved the study protocol (KB-0012/46/02/19).

\section{Statistical analysis}

To compare the characteristics of NVAF stroke patients with stroke patients without AF, we used the Mann-Whitney test (quantitative data) and the Fisher exact test (qualitative data). In addition, univariable and multivariable logistic regression models were calculated for assessing the odds ratio of chosen risk factors of AF incidence among IS patients. Statistical significance was set at $p<0.05$.

\section{Results}

Comparison of risk factors for atrial fibrillation in patients with ischemic stroke and nonvalvular atrial fibrillation and patients with ischemic stroke without atrial fibrillation

Cigarette smoking, hypertension, older age, diabetes mellitus, female gender, dyslipidemia, coronary heart disease, history of ischemic stroke, and higher $\mathrm{CHA}_{2} \mathrm{DS}_{2}$-VASc score were significantly more frequent in patients with IS and NVAF than in patients without $\mathrm{AF}(p<0.01, p<0.01, p<0.01$, $p<0.01, p<0.01, p<0.01, p<0.01, p<0.01$, $p<0.01)$. A case comparison is presented in Table I.

\section{Analysis of risk factors for AF in patients who suffered an ischemic stroke}

In this study we found - based on a univariable and multivariable logistic regression model - that compared to the patients with IS without AF, the group of patients who suffered from IS with NVAF had a higher proportion of patients who smoked cigarettes $(\mathrm{OR}=15.742, p<0.01 ; \mathrm{OR}=41.1$, $p<0.01)$, had hypertension ( $\mathrm{OR}=5.161, p<0.01$; $\mathrm{OR}=5.666, p<0.01)$, history of a previous stroke $(\mathrm{OR}=3.951, p<0.01 ; \mathrm{OR}=4.792, p<0.01)$, dyslipidemia $(\mathrm{OR}=2.312, p<0.01 ; \mathrm{OR}=1.592$, $p<0.01)$, coronary heart disease $(\mathrm{OR}=2.306$, $p<0.01 ; \mathrm{OR}=1.988, p<0.01)$, a greater proportion of female patients $(\mathrm{OR}=1.717, p<0.01$; $\mathrm{OR}=2.095, p<0.01)$, higher incidence of diabetes mellitus $(\mathrm{OR}=1.341, p<0.01 ; \mathrm{OR}=1.261$, $p=0.106)$ and more patients in old age (OR = 1.084, $p<0.01 ; \mathrm{OR}=1.101, p<0.01$ ) (Table II).

\section{Discussion}

According to our analysis, stroke patients with concurrent AF were significantly older $(p<0.01)$, more frequently female $(p<0.01)$, and significantly more frequently $(p<0.01)$ had type 2 diabe- 
Table I. Characteristics of patients with ischemic stroke. Patients with NVAF vs. patients without AF

\begin{tabular}{|c|c|c|c|}
\hline Parameter & $\begin{array}{c}\text { Group A (NVAF) } \\
\quad(n=696)\end{array}$ & $\begin{array}{l}\text { Group B (no AF) } \\
\quad(n=1678)\end{array}$ & $P$-value \\
\hline Age & $77.05 \pm 9.88$ & $67.5 \pm 11.52$ & $<0.01$ \\
\hline \multicolumn{4}{|l|}{ Sex: } \\
\hline Females & $446(18.8 \%)$ & $855(36.0 \%)$ & \multirow[t]{2}{*}{$<0.01$} \\
\hline Males & $250(10.5 \%)$ & $823(34.7 \%)$ & \\
\hline \multicolumn{4}{|c|}{ Diabetes mellitus: } \\
\hline No & $438(18.4 \%)$ & $1166(49.1 \%)$ & \multirow[t]{2}{*}{$<0.01$} \\
\hline Yes & 258 (10.9\%) & $512(21.6 \%)$ & \\
\hline \multicolumn{4}{|l|}{ Dyslipidemia: } \\
\hline No & $340(14.3 \%)$ & 1155 (48.7\%) & \multirow[t]{2}{*}{$<0.01$} \\
\hline Yes & $356(15.0 \%)$ & $523(22.0 \%)$ & \\
\hline \multicolumn{4}{|l|}{ Hypertension: } \\
\hline No & $57(2.4 \%)$ & $529(22.3 \%)$ & \multirow[t]{2}{*}{$<0.01$} \\
\hline Yes & $639(26.9 \%)$ & 1149 (48.4\%) & \\
\hline \multicolumn{4}{|c|}{ Congestive heart failure: } \\
\hline No & $604(25.4 \%)$ & $1426(60.1 \%)$ & \multirow[t]{2}{*}{0.28} \\
\hline Yes & $92(3.9 \%)$ & $252(10.6 \%)$ & \\
\hline \multicolumn{4}{|c|}{ Coronary heart disease: } \\
\hline No & $261(11.0 \%)$ & $974(41.0 \%)$ & \multirow[t]{2}{*}{$<0.01$} \\
\hline Yes & $435(18.3 \%)$ & $704(29.7 \%)$ & \\
\hline \multicolumn{4}{|l|}{ Smoker: } \\
\hline No & $196(8.3 \%)$ & $1444(60.8 \%)$ & \multirow[t]{2}{*}{$<0.01$} \\
\hline Yes & $500(21.1 \%)$ & 234 (9.9\%) & \\
\hline \multicolumn{4}{|c|}{ Previous stroke: } \\
\hline No & 377 (15.9\%) & $1382(58.2 \%)$ & \multirow[t]{2}{*}{$<0.01$} \\
\hline Yes & $319(13.4 \%)$ & $296(12.5 \%)$ & \\
\hline $\mathrm{CHA}_{2} \mathrm{DS}_{2}-\mathrm{VASC}$ & $5.39 \pm 0.91$ & $2.95 \pm 1.16$ & $<0.01$ \\
\hline
\end{tabular}

tes, dyslipidemia, arterial hypertension, coronary heart disease, smoked tobacco products, and had a history of additional prior ischemic stroke, compared to stroke patients without AF. Patients with AF significantly more frequently manifested features which, according to our study, are not only independent risk factors for stroke, but most likely also increase risk of AF. Based on uni- and multivariable regression, the risk of atrial fibrillation among ischemic stroke patients is particularly high in patients with hypertension, stroke history, dyslipidemia, coronary heart disease, type 2 diabetes, older patients, smokers, and women.

Smoking is a well-known risk factor for cardiovascular diseases [17]. Nicotine stimulates a sig- nificant release of catecholamines into the serum, thus increasing the risk of cardiac arrhythmias. A study by Goette et al. concluded that exposure of healthy atrial tissues to nicotine caused a 10fold increase in collagen III at the mRNA level, comparable to that observed in smokers [18]. The resulting atrial fibrosis is a central mechanism in the development of AF.

Arterial hypertension related to dysfunction of the renin-angiotensin-aldosterone system is associated with left ventricular hypertrophy, impaired left ventricular diastolic filling, increased left atrial pressure, atrial fibrosis, increased atrial ectopic activity, and a reduced rate of electrical conduction in and between atria [19]. Epidemiological studies 
Table II. Analysis of risk factors for atrial fibrillation in patients with non-valvular atrial fibrillation who suffered a stroke compared to patients without AF (multivariate logistic regression)

\begin{tabular}{|c|c|c|c|c|c|c|}
\hline \multirow[t]{2}{*}{ Factors } & \multicolumn{3}{|c|}{ Univariable logistic regression models } & \multicolumn{3}{|c|}{ Multivariable logistic regression model ${ }^{*}$} \\
\hline & OR & $\mathrm{Cl}$ & $P$-value & OR & $\mathrm{Cl}$ & $P$-value \\
\hline Age & 1.084 & $1.074-1.094$ & $<0.01$ & 1.101 & $1.087-1.117$ & $<0.01$ \\
\hline \multicolumn{7}{|c|}{ Hypertension: } \\
\hline Yes & 5.161 & $3.895-6.962$ & $<0.01$ & 5.666 & $3.839-8.521$ & $<0.01$ \\
\hline No & 1.0 & - & - & 1.0 & - & - \\
\hline \multicolumn{7}{|l|}{ Smoker: } \\
\hline Yes & 15.742 & $12.719-19.567$ & $<0.01$ & 41.1 & $29.667-57.914$ & $<0.01$ \\
\hline No & 1.0 & - & - & 1.0 & - & - \\
\hline \multicolumn{7}{|c|}{ Previous stroke: } \\
\hline Yes & 3.951 & $3.253-4.804$ & $<0.01$ & 4.792 & $3.582-6.446$ & $<0.01$ \\
\hline No & 1.0 & - & - & 1.0 & - & - \\
\hline \multicolumn{7}{|c|}{ Dyslipidemia: } \\
\hline Yes & 2.312 & $1.93-2.772$ & $<0.01$ & 1.592 & $1.217-2.084$ & $<0.01$ \\
\hline No & 1.0 & - & - & 1.0 & - & - \\
\hline \multicolumn{7}{|c|}{ Congestive heart failure: } \\
\hline Yes & 0.862 & $0.664-1.111$ & 0.257 & 0.758 & $0.454-1.252$ & 0.284 \\
\hline No & 1.0 & - & - & 1.0 & - & - \\
\hline \multicolumn{7}{|l|}{ Sex: } \\
\hline Females & 1.717 & $1.433-2.062$ & $<0.01$ & 2.095 & $1.574-2.803$ & $<0.01$ \\
\hline Males & 1.0 & - & & 1.0 & - & - \\
\hline \multicolumn{7}{|c|}{ Diabetes mellitus: } \\
\hline Yes & 1.341 & $1.114-1.614$ & $<0.01$ & 1.261 & $0.952-1.671$ & 0.106 \\
\hline No & 1.0 & - & - & 1.0 & - & - \\
\hline \multicolumn{7}{|c|}{ Coronary heart disease: } \\
\hline Yes & 2.306 & $1.924-2.767$ & $<0.01$ & 1.988 & $1.511-2.623$ & $<0.01$ \\
\hline No & 1.0 & - & - & 1.0 & - & - \\
\hline
\end{tabular}

${ }^{\star}$ Full adjusted model; Independent variables: age; hypertension (yes/no); smoker (yes/no); previous stroke (yes/no); dyslipidemia (yes/ no); congestive heart failure (yes/no); sex (F/M); diabetes mellitus (yes/no); coronary heart disease (yes/no). Group A (Status = 1) - NVAF. Group B (Status = 0) - No NVAF.

show that arterial hypertension is associated with a 1.8 times higher risk of $\mathrm{AF}$ and a 1.5 times higher risk of AF progression, compared to chronic unchanging $A F[7,20]$. Although as yet no randomized studies have conclusively shown that hypotensive therapy reduces the risk of $\mathrm{AF}$, studies do suggest that effective hypertension treatment, especially renin-angiotensin-aldosterone antagonists, may reduce the likelihood of developing AF [21].

Coronary heart disease is both a risk factor for the development of AF and a disease whose progress is modulated by AF. The presence of coronary heart disease in patients with previously diagnosed atrial fibrillation has been associated with recurrent episodes of $\mathrm{AF}$ and a substantially increased risk of mortality [22].

The risk of $A F$ increases with age, and the condition is much less common in people under 50 years of age. After the sixth decade of life, the incidence of AF increases significantly, from 0.5\% in people aged over 50 to almost $9 \%$ in the eighth decade of life [4, 23]. It is not surprising, therefore, that among our patients, age is not only a risk factor for stroke, but also for AF. The incidence of AF in all age groups is higher in men. However, our study found that the incidence of atrial fibrillation 
was higher among women. Females with AF have a higher stroke risk than males with AF [24]. This could be a reason for the higher incidence of $A F$ in this study of stoke patients.

Diabetes mellitus is one of the leading chronic diseases in patients with AF. For many years, diabetes mellitus was not taken as a risk factor for developing AF. The view on DM and AF changed in the last decade of the previous century. As shown in the Framingham study, diabetes is an independent risk factor for atrial fibrillation with an OR of 1.6 for women and 1.4 for men. Carbohydrate metabolism disorders have a direct damaging effect on the myocardium, promoting the development of AF [7].

Dyslipidemia is a major risk factor for cardiovascular disease. The association between dyslipidemia and AF, however, has been controversial. One study showed that hypercholesterolemia was more frequent in the non-AF group than in the AF group [25]. In another study low serum levels of total cholesterol, triglyceride and high-density lipoprotein cholesterol (HDL-C) levels were found in patients with paroxysmal AF [26]. In another cross-sectional study low levels of HDL-C were associated with the development of AF in women. This study also found that women have a $28 \%$ higher risk of developing AF with every $10 \%$ decrease in $\mathrm{HDL}-\mathrm{C}$. There was no correlation between TG levels and AF [27]. There are some potential mechanisms by which dyslipidemia is associated with AF and our study also confirms this. Elevated LDL and decreased levels of HDL cholesterol are associated with reduced systolic and diastolic left ventricle function and the development of heart failure, which are risk factors for AF [28]. Inflammation and oxidative stress are strongly associated with dyslipidemia. Both of them elevate the susceptibility to $\operatorname{AF}[29,30]$. The results of the meta-analysis carried out by Wang et al. and including twenty randomized controlled trials showed that statin therapy is useful for the prevention of AF [31].

$\mathrm{CHA}_{2} \mathrm{DS}_{2}$-VASc score is a scale used to assess the risk of thromboembolic complications in patients with AF. It is an improved $\mathrm{CHADS}_{2}$ score, augmented with additional risk factors of thromboembolic complications. Its individual components not only predict the risk of stroke associated with $A F$, but are also associated with the development of AF. In several studies this score has been used to predict the presence of AF in patients with ischemic stroke, although the results were inconsistent [32, 33]. Liu et al. in their study observed that among all risk factors, including the $\mathrm{CHA}_{2} \mathrm{DS}_{2}$ VASc score, only age and circulatory insufficiency were independently related to the newly detected AF in patients with acute IS [34]. In our study, patients with IS and coexisting AF had significantly higher $\mathrm{CHA}_{2} \mathrm{DS}_{2}$-VASc scores. This finding is not surprising because the $\mathrm{CHA}_{2} \mathrm{DS}_{2}$-VASc score takes into account causal risk factors for $A F$, and in our study patients with IS and co-existing AF were characterized by significantly more frequent $A F$ risk factors such as hypertension, older age, diabetes mellitus, female gender, coronary heart disease and history of ischemic stroke.

Our study had several limitations. This was a single-center retrospective study, with a limited number of stroke patients. We selected only the most common atrial fibrillation risk factors. The participants in our 'IS with no NVAF' group may have undiagnosed AF. Further prospective studies with a larger sample size are needed.

In conclusion, it is not surprising that ischemic stroke patients with non-valvular atrial fibrillation - which is considered to be the primary risk factor for a cerebral episode - also display other risk factors. It should be emphasized, however, that in the examined group of patients, the most important stroke risk factors were significantly more closely associated with the concurrent AF. This was especially true for smoking, hypertension, dyslipidemia, coronary heart disease, and diabetes mellitus. Therefore, it should be assumed that these may be independent risk factors not only for stroke, but also for AF. This interpretation is particularly salient from a neurological point of view. The role of AF as an important risk factor for ischemic stroke remains underestimated, possibly due to the difficulty of detecting AF in its paroxysmal presentation. Therefore, it is necessary to perform long-term monitoring of cardiac function (e.g. Holter) to detect this arrhythmia.

Our study demonstrates a need for thorough and systematic monitoring of post-ischemic stroke patients in whom AF has not been detected and who display other important risk factors. Regardless of the stroke, these factors may be responsible for development of AF, and therefore it should be assumed that these patients have yet undiagnosed but already existent paroxysmal AF, or that their risk of developing AF is particularly high. This is important due to the resulting need to prescribe anticoagulants for the prevention of subsequent cerebral vascular episodes, as opposed to the standard antiaggregant drugs used for the prevention of ischemic stroke in the majority of cases.

\section{Conflict of interest}

The authors declare no conflict of interest.

\section{References}

1. Chugh SS, Havmoeller R, Narayanan K, et al. Worldwide epidemiology of atrial fibrillation: a Global Burden of Disease 2010 Study. Circulation 2014; 129: 837-47. 
2. Fatkin D, Kelly RP, Feneley MP. Relations between left atrial appendage blood flow velocity, spontaneous echocardiographic contrast and thromboembolic risk in vivo. J Am Coll Cardiol 1994; 23: 961-9.

3. Krijthe BP, Kunst A, Benjamin EJ, et al. Projections on the number of individuals with atrial fibrillation in the European Union, from 2000 to 2060. Eur Heart J 2013, 34: 2746-51.

4. Go AS, Hylek EM, Phillips KA, et al. Prevalence of diagnosed atrial fibrillation in adults: national implications for rhythm management and stroke prevention: the AnTicoagulation and Risk Factors in Atrial Fibrillation (ATRIA) Study. JAMA 2001; 285: 2370-5.

5. Dublin S, Glazer NL, Smith NL, et al. Diabetes mellitus, glycemic control, and risk of atrial fibrillation. J Gen Intern Med 2010; 25: 853-8.

6. Wilhelmsen L, Rosengren A, Lappas G. Hospitalizations for atrial fibrillation in the general male population: morbidity and risk factors. J Intern Med 2001; 250: 382-9.

7. Benjamin EJ, Levy D, Vaziri SM, et al. Independent risk factors for atrial fibrillation in a population-based cohort: the Framingham Heart Study. JAMA 1994; 271 840-4.

8. Mejias S, Ramphul K. Prevalence of peripheral arterial disease among diabetic patients in Santo Domingo, Dominican Republic and associated risk factors. Arch Med Sci Atheroscler Dis 2018; 3: 35-40.

9. Zemtsovskaja G, Abina J, Meigas K, Pilt K, Viigimaa M. Pulse wave velocity and its gender-related associations with cardiovascular risk factors in a high cardiovascular risk population. Arch Med Sci Atheroscler Dis 2018; 3 : e99-105.

10. Wańkowicz P, Nowacki P, Gołąb-Janowska M. Risk fac tors for ischemic stroke in patients with non-valvular atrial fibrillation and therapeutic international normalized ratio range. Arch Med Sci 2018. doi:10.5114/ aoms.2018.77546.

11. Hariri E, Hachem A, Sarkis G, Nasr S. Optimal duration of monitoring for atrial fibrillation in cryptogenic stroke: a nonsystematic review. Biomed Res Int 2016; 2016 5704963.

12. Brambatti M, Connolly SJ, Gold MR, et al. Temporal relationship between subclinical atrial fibrillation and embolic events. Circulation 2014; 129: 2094-9.

13. Pandey A, Garg S, Khunger M, et al. Dose-response relationship between physical activity and risk of heart failure: a meta-analysis. Circulation 2015; 132: 1786-94.

14. Tahrani AA, Bailey CJ, Del Prato S, Barnett AH. Management of type 2 diabetes: new and future developments in treatment. Lancet 2011; 378: 182-97.

15. Grundy SM, Cleeman JI, Merz CN, et al. Implications of recent clinical trials for the National Cholesterol Education Program Adult Treatment Panel III guidelines. Arterioscler Thromb Vasc Biol 2004; 24: e149-61.

16. Mancia G, De Backer G, Dominiczak A, et al. Management of Arterial Hypertension of the European Society of Hypertension/European Society of Cardiology. 2007 Guidelines for the Management of Arterial Hypertension: The Task Force for the Management of Arterial Hypertension of the European Society of Hypertension (ESH) and of the European Society of Cardiology (ESC). J Hypertens 2007; 25: 1105-87.

17. Yanbaeva DG, Dentener MA, Creutzberg EC, Wesseling G, Wouters EF. Systemic effects of smoking. Chest 2007; 131: 1557-66.
18. Goette A, Lendeckel U, Kuchenbecker A, et al. Cigarette smoking induces atrial fibrosis in humans via nicotine. Heart 2007; 93: 1056-63.

19. Go O, Rosendorff C. Hypertension and atrial fibrillation. Curr Cardiol Rep 2009; 11: 430-5.

20. De Vos CB, Pisters R, Nieuwlaat $R$, et al. Progression from paroxysmal to persistent atrial fibrillation clinical correlates and prognosis. J Am Coll Cardiol 2010; 55: 725-31.

21. Healey JS, Baranchuk A, Crystal E, et al. Prevention of atrial fibrillation with angiotensin-converting enzyme inhibitors and angiotensin receptor blockers: a metaanalysis. J Am Coll Cardiol 2005; 45: 1832-9.

22. Murakami N, Tanno M, Kokubu N, et al. Distinct risk factors of atrial fibrillation in patients with and without coronary artery disease: a cross-sectional analysis of the BOREAS-CAG Registry data. Open Heart 2017; 4: e000573.

23. Furberg CD, Psaty BM, Manolio TA, Gardin JM, Smith VE, Rautaharju PM. Prevalence of atrial fibrillation in elderly subjects (the Cardiovascular Health Study). Am J Cardiol 1994; 74: 236-41.

24. Michelena HI, Powell BD, Brady PA, Friedman PA, Ezekowitz MD. Gender in atrial fibrillation: ten years later. Gend Med 2010; 7: 206-17.

25. Iguchi Y, Kimura K, Aoki J, et al. Prevalence of atrial fibrillation in community-dwelling Japanese aged 40 years or older in Japan: analysis of 41,436 non-employee residents in Kurashiki-city. Circ J 2008; 72: 909-13.

26. Annoura M, Ogawa M, Kumagai K, Zhang B, Saku K, Arakawa K. Cholesterol paradox in patients with paroxysmal atrial fibrillation. Cardiology 1999; 92: 21-7.

27. Watanabe H, Tanabe N, Yagihara N, Watanabe T, Aizawa Y, Kodama M. Association between lipid profile and risk of atrial fibrillation. Circ J 2011; 75: 2767-74.

28. Velagaleti RS, Massaro J, Vasan RS, et al. Relations of lipid concentrations to heart failure incidence: the Framingham Heart Study. Circulation 2009; 120: 2345-51.

29. Chung MK, Martin DO, Sprecher D, et al. C-reactive protein elevation in patients with atrial arrhythmias: inflammatory mechanisms and persistence of atrial fibrillation. Circulation 2001; 104: 2886-91.

30. Mihm MJ, Yu F, Carnes CA, et al. Impaired myofibrillar energetics and oxidative injury during human atrial fibrillation. Circulation 2001; 104: 174-80.

31. Wang Z, Zhang Y, Gao M, et al. Statin therapy for the prevention of atrial fibrillation: a meta-analysis of randomized controlled trials. Pharmacotherapy 2011; 31: 1051-62.

32. Ziegler PD, Glotzer TV, Daoud EG, et al. Incidence of newly detected atrial arrhythmias via implantable devices in patients with a history of thromboembolic events. Stroke 2010; 41: 256-60.

33. Favilla CG, Ingala E, Jara J, et al. Predictors of finding occult atrial fibrillation after cryptogenic stroke. Stroke 2015; 46: 1210-5.

34. Liu R, Yang X, Li S, Jiang Y, Wang Y, Wang Y. Novel composite scoring system to predict unknown atrial fibrillation in acute ischemic stroke patients. Brain Res 2017; 1674: 36-41. 